\title{
SOME REMARKS ON THE NATURE AND TREATMENT OF STRABISMUS
}

\author{
BY \\ Dr. Delogé, \\ NICE, FRANCR
}

EX-ASSISTANT IN OPHTHALMOLOGY IN THE PARIS HOSPITALS

THE works of Donders, Javal, and Parinaud have profoundly modified our conception of strabismus. They have, in their turn, reviewed the therapeutics of this affection.

One of the first disciples of Dr. Rémy, the author of the Diploscope, has pleasure in putting forward, in this paper, for the benefit of its numerous readers, a few new views concerning binocular vision, strabismus and its treatment.

Rémy's diploscope has become indispensable to the oculist. It is the real instrument of control in the measurements of the frequent alterations of binocular vision. Most simple in construction and consisting of a long rod, $1.20 \mathrm{~m}$. in length, carrying in its centre a plate, perforated with two apertures, and at its end a cardboard, marked with letters or coloured.

The slightest alterations in binocular vision are immediately recognised by the displacements of the letters, which no longer appear in their normal place, but as in crossed diplopia if there be divergent strabismus, or as in homonymous diplopia if there be convergent strabismus.

To facilitate the understanding of the working of the instrument, let us experiment with four letters. The cases coming before our notice being the following :-

(1.) Binocular vision being normal the results will be :-

$$
\mathrm{K} \quad \mathrm{O} \quad \mathrm{L} \quad \mathrm{A}
$$

(2.) Neutralization of one eye, and, depending upon the eye involved :-

$$
\text { O A or } \mathbf{K} \quad \mathbf{L}
$$

(3.) Should there be divergence the patient will read :-

$$
\mathbf{K} \text { OL } \mathbf{A}
$$

(or) If the degree be accentuated:-

$$
\begin{aligned}
& \begin{array}{lll}
\mathbf{O} & \mathbf{A}
\end{array} \\
& \text { K L } \quad \text { O } \\
& \text { K LO A } \\
& \text { K L O A } \\
& \text { KL OA etc. }
\end{aligned}
$$


(4.) Should there be convergence the reading will be :-

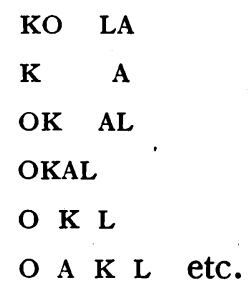

(5.) Lastly, should there be sursum or deorsumvergence the letters will place themselves on different lines :-

$$
{ }^{\mathrm{K}}{ }^{\mathrm{L}} \mathrm{A}
$$

with or without horizontal strabismus trouble.

The definition of "strabismus" as given by Parinaud is very explicit. It is a question of " a flaw in the development of the binocular sight apparatus, preventing the convergence of the eyes upon a fixed object." It is a central discipline disorder. This conception explains certain cases of "strabismus" where the optical causes are non-apparent, bringing out the importance of personal morbid accidents (infectious diseases), or hereditary defect (syphilis, alcoholism, consanguinity).

Most children suffering from "strabismus" are neurotic subjects, and "strabismus" develops on different lines in each special case.

However, no theory is absolutely correct, and, however true that of Parinaud may seem, in so far as it concerns strabismus in children, it does not seem to apply to the habitual strabismus in the adult.

Our intention is to show precisely that this affection can supervene among individuals whose binocular sight is perfectly developed, and without necessarily being connected with special nervous influences. Here the visual alteration alone is at fault.

How does " strabismus " evolve?

Strabismus must not be confounded with an apparent deviation or squint, which may not really exist (invisible strabismus), or again, with paralysis. The first degree of "strabismus" is simultaneous sight. The two eyes see at the same time notwithstanding a slight inco-ordination, whence may come visual uneasiness.

This period is soon succeeded by partial simultaneous sight (simultaneous sight of Parinaud), which we have elsewhere called panoramic vision. Parinaud has defined it very well in further naming it "alternating central vision." Neutralization is the most typical and curious characteristic of this period. 
In panoramic sight, neutralization is the reaction of the defence against diplopia, and the more rapidly it intervenes the less the patient suffers from the inconvenience of the preceding period.

This neutralization is elective and keeps exclusively to that portion of the retina corresponding to the object seen by the nondeviating eye and avoiding the other regions of the visual field.

Neutralization does not always follow the same lines. It is alternating and generally remains so when both eyes are of equal strength or when one is hypermetropic and the other myopic.

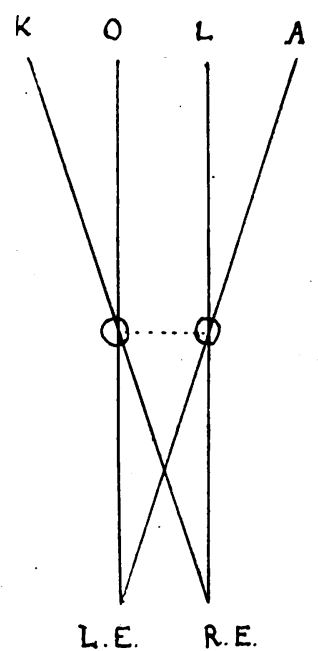

Fig. 1.

Illustration of the four-letter experiment for normal eyes $\mathrm{KL}$ is seen by the right eye. $\mathrm{O}$ A by the left.

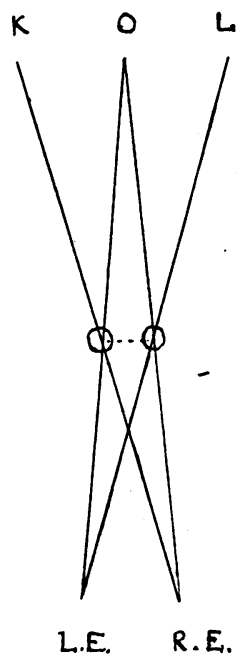

Fig. 2.

Scheme of the three-letter experiment for normal eyes. $\mathrm{KO}$ is seen by the right eye. $\mathrm{O} L$ is seen by the left.*

But at times, with or without appreciable cause, where one of the eyes is manifestly inferior to the other, neutralization becomes fixed, and alternatively the sight becomes monocular. This new stage is the most serious on account of the momentous disorders it brings about. One of the most embarrassing of these accidents is amblyopia ex anopsia.

Simultaneous sight, alternating, central, or panoramic and monocular are the stepping stones, the successive and characteristic stages of strabismus. In the course of this affection amblyopia and deviation itself are but frequent, simple and, often, very annoying disorders.

Strabismus in the adult.-An appreciable difference in the sight of the eyes is the usual cause of strabismus in the adult. Hering compares the optical apparatus to a double team driven by single

"Ch. Delogé.- " On Corrections of Anisometropia," Arch. d'Ophtalmologie, 1904. 
reins. We will use his ingenious idea. If one of the team goes lame, it either stumbles on, or stops, and must be detached. It is the same case with the sight. The causes of strabismus in the adult are, then, very varied. I have recounted thirty cases of anisometropia, chosen from many others, in my inaugural treatise* : each time the difference of refraction exceeded two dioptres, a visual alteration, an actual strabismus existed before correction.

The onset of strabismus in the adult, as in the child, is slow and progressive, and of insidious growth.

Particular characteristics.-Deviation, apparent or not, is nearly always produced from outside, and divergent strabismus is much more frequent. Amblyopia ex anopsia is less pronounced. Strabismus is more frequently seen among perfectly balanced and healthy individuals with whom the affection is ocular, and purely accidental. Such form of strabismus is very easy to cure.

Treatment.-We shall say nothing on the operative treatment. It is the only treatment to be resorted to in two circumstances :When the visual acuteness of one of the eyes is definitely bad or far inferior to the other, or when it is impossible to secure the necessary attendance of the subject.

In all other circumstances we think that the orthoptic treatment is preferable, not only because it is the most reasonable, but because it is the only one to give complete results.

Convergent strabismus.-Convergent strabismus is particularly frequent in children, but its origin is not always recognized as the same. It is very often allied with a nervous central disorder (Parinaud); at times the influence of refraction is shown (Donders); and in other cases these two causes mingle so closely that it is impossible to say which part is due to the nervous system and for which abnormal refraction is responsible.

In the first instance a most careful and minute examination is necessary as well as optical correction. By these means many cases of strabismus improve spontaneously. If there is amblyopia we must not be satisfied with an improved squint, but make the amblyopic eye work most actively so as to gain time. Just as soon as the visual acuteness reaches two or three tenths, commence exercises to re-establish binocular sight.

Each one, in their turn, the stereoscope and the amblyoscope have been well spoken of. These instruments certainly possess certain qualities as well as certain faults which interfere with perfect re-education. It is the diploscope, large size or small, to which we must turn.

The first thing to be obtained is simultaneous sight, diplopia. This is generally very rapidly obtained by a little ingeniousness. Neutralization often gives way in showing the letters in a vertical

*"Anisometropia and Binocular Sight." Paris, 1907. 
position or in replacing those that are not seen by colours, brilliant objects, etc.

The great, or shall we say, the greatest obstacle or difficulty is to obtain divergent movement. The difficulty is reduced by the use of prisms of weaker and weaker strengths.

If the child is willing and keen, and the parents are patient, it will be noticed that the amblyopia will improve and a cure will follow. Even where these means fail to effect a cure, the exercises prepare the ground in a wonderful manner for surgical intervention. Nevertheless, the most perfect operation must always be completed by diploscopic exercise as well as by perfect optical correction in order to maintain results.

Divergent strabismus.- Most oculists appear to think that this variety of strabismus, however accentuated the case may be, requires surgical intervention. Outside the case of amblyopia nearly incurable in one eye, the contrary seems nearer the truth.

Let us say at once that one often meets with an appreciable difference of sight at the outset, resulting from lesion (leucoma or monocular cataract), or again through anisometropia caused by myopia or by unilateral astigmatism.

The hundreds of cases that have come before our notice permit us to affirm that when the degree of anisometropia has surpassed two dioptres there is generally suppression of normal binocular sight, and, in many cases, apparent divergent strabismus. A marked anisometropia is, in itself alone, sufficient reason; of this I am certain from personal experience. I have $5 \mathrm{D}$ of myopia in both eyes, and after 15 or 20 days of monocular correction the annoyance caused during the first few days disappeared, but monocular sight and an apparent divergence had been established in the uncorrected eye.

Generally, oculists have quickly given up the correction, which far from improving the sight, became a source of inconveniences, such as vertigo, nervous headache, false relief and dizziness. Taught by these facts, practitioners have wisely dissuaded the correction of anisometropia superior to one dioptre and a half.

In our turn we have resumed this study, aided by Rémy's diploscope, and found reasons to oppose a full correction. These reasons are two in number:-Strabismic deviation - the cause of more or less obvious diplopia which is often sufficient to awaken improved visual acuteness, and the unequal size of objects seen by each eye. Many years of experience convince us that the inequality in the size of objects, which is such a trouble to the patient in the beginning, never persists. It is not so with deviation, this requires a more or less lengthy treatment, and only yields to a course of suitable exercises.

The real difficulty arises from the insufficient degree of conver- 
gence; that it is which causes all delays in cure, therefore our first effort ought to be that of diminishing and vanquishing it. Convergence once established, victory is at hand, for the re-education of binocular sight becomes henceforward, rapid and easy.

We will rapidly sum up our usual course of treatment in the greater number of cases of divergent strabismus.

In the first place, full correction of the ametropia, no matterwhat the degree or how marked the difference in refraction between the two eyes; I have even corrected and cured two patients suffering from unilateral aphakia, details of which have been published in my thesis. If this correction is not sufficient to remove neutralization, and this happens frequently where the deviation is very pronounced, I try to revive diplopia. Many excellent means may be utilised to this end, either simultaneously or separately. Diploscopic exercises, coloured glasses and different degrees of light will often suffice. In rebellious cases, prisms must be resorted to. I have used seventy dioptres of prisms in the case of one of my patients (Dr. G.).

In a very few rare cases, when the front sight was good, perimeter exercises were necessary to complete the cure of lateral sight. If the convergence is good and the subject willing the result is more rapidly obtained than by an operation.

A few years ago I cured a young man in twelve sittings (Mr. G. de S. J.) suffering from considerable divergent strabismus due to unilateral myopia of six dioptres.

I will briefly mention the following case of Mlle. E- Maged 17 .

I saw this patient on August 2, 1904, at the Hotel-Dieu. She presented a paracentral leucoma of the right eye and myopic astigmatism.

Skiascopy :-R.E. (vertical axis -2 D., horizontal axis -6 D.), L.E. (emmetropic).

Ophthalmometer :-R.E. $=5$ D. As. L.E. = Normal.

The sight of the right eye after correction equalled 0.5 only. This girl squinted very markedly outwards and a little above, but this deviation was not visible at first with the large diploscope, and it took half an hour of rather trying exercises to make the neutralization, which was alternating, disappear. Her sight soon became simultaneous, and the diploscope revealed a divergent and sursumvergent strabismus, of the existence of which I already felt sure. The next day her sight was still simultaneous, and the letters seen by the right eye, armed with its correction, appeared much smaller. I diminished the strength of the spherical glass by $1.5 \mathrm{D}$., and immediately the letters became equal. Two days later I returned to my first correction :-R.E. (horizontal axis -5 D. sph. - 6 D. cyl.) and the letters remained the same size. The sight of 
the right eye is now 0.9. The binocular sight is normal. In this case, exercises for six days sufficed to cure a marked ametropia, complicated by astigmatism, and a double deviation, one of which was quite considerable. Seen two months later, the sight of Miss M-_ remained perfect.

\title{
Conclusion
}

Owing to its double nervous and optical origin, convergent strabismus requires a treatment that is lengthy and particularly difficult to establish, but in which re-education of binocular vision is essential. Where possible this constitutes the preferable method, as well as the indispensable complement to surgical intervention. We ought to beware of the opinion of a great number of excellent oculists who believe divergent strabismus can only be relieved by surgery. With some reservations we hold the contrary to be the truth, and it is chiefly in this case that orthoptic treatment gives the most rapid results and brilliant successes.

\section{GLIOMA RETINAE (WITH REPORT OF A CASE)}

BY

\author{
Dr. M. J. KeYS \\ VICTORIA, B.c.
}

True glioma retinae is seen so rarely and the subject is so interesting that it warrants publication of the details of every case observed.

The following case recently occurred in my own practice :-

Case History.-J. D., female, was born in the Jubilee Hospital, Victoria, two and a half years ago. She is the only child in the family. Mother pregnant but once. Parents : father, English ; his mother was Scotch. The mother of the child is English, born in Lancashire; no history of any similar eye trouble on either side of the house. The little patient when born was a healthy baby, labour was normal, and she has never had a day's illness in her life, not even the diseases common to childhood, and when first seen by me, September 28, 1920, was a well-nourished, healthy child.

History of present illness.-About two weeks previously, a lady, one of the neighbours, drew the attention of the mother to the peculiar appearance of the child's right eye, and about the same time the child's cousin, a lad of 12 years of age, noticed the same thing, and curiously enough, remarked that it looked like a " cat's 